\title{
The transport properties of cement mortars subjected to freeze-thaw cycles
}

\author{
Alicja Wieczorek ${ }^{1, *}$, Marcin Koniorczyk $^{1}$, Dalia Bednarska $^{1}$, Kalina Grabowska $^{1}$ \\ ${ }^{1}$ Department of Building Physics and Building Materials, Lodz University of Technology, 90-924 \\ Lodz, Al. Politechniki 6, Poland
}

\begin{abstract}
The parameters characterizing the microstructure of cementbased materials, such as porosity or permeability, determine not only durability, but also risk of degradation of the cement matrix due to an aggressive environment. The report presents results of a research on transport properties of cement mortars subjected to cyclic water freezing. Mortars prepared on the basis of two different cements were the object of the research: Portland cement CEM I 42.5R and Portland blast-furnace slag cement CEM III/A 42.5N LH/HSR/NA, with two water-cement ratio $(\mathrm{w} / \mathrm{c}=0.45$ and 0.40$)$. The experimental study was carried out in order to determine the relationship between intrinsic permeability and the water absorption coefficient in relation to the number of freeze-thaw cycles. The evolution of transport coefficients was determined using a capillary absorption test and the modified RILEM-Cembureau method. It was established that the degradation processes induced an increase of transport properties. Moreover, the microcracks had a more significant influence on permeability and lesser influence on the water absorption coefficient. The gas permeability of damaged mortar changed very significantly, an increase with several orders of magnitude could be noticed. Moreover, the positive impact of CEM III on ice-induced degradation was also visible.
\end{abstract}

\section{Introduction}

The durability and frost resistance of cement-based material are directly influenced by the transport properties of the porous matrix. The amount of a fluid which can penetrate into a porous structure highly influences the range of cement matrix degradation [1]. The porous materials are built of a solid skeleton and pores (different types of pores with different pore sizes), which might be filled with the gaseous and the liquid phase. The liquid phase contains, besides water, various types of contaminations, which migrate into the material through the system of interconnected pores.

Micro-cracking induced by frost action tends to connect the capillary pores. It can be reflected as a change in the microstructure of cement-based materials. Therefore, the microstructure is one of the most important characteristics, which estimates the durability of cement based materials [2]. Frost degradation of the cement matrix causes

\footnotetext{
*Corresponding author: alicja.marciniak@p.lodz.pl
} 
a redistribution of pore sizes [3]. In particular, it is associated with an increased volume of pores with diameters larger than $100 \mathrm{~nm}$. The degradation of the porous building material can significantly alter its transport properties, adversely affecting the durability of structures. Therefore, in order to account for the effect of damage on the designed service life of structures, its impact on mass transport need to be taken into consideration. The transport properties are related to the pore size distribution and interconnectivity of the pore system of cement-based materials. Furthermore, the transport properties are adversely affected by the presence of cracks. The influence of pore size on the permeability of concrete is significant, pores $>100 \mathrm{~nm}$ can obviously increase the permeability of concrete [4]. The relationship between permeability and microstructure of concrete were investigated by [5]. According to obtained results, the gas permeability coefficient has a high correlation with the contributive porosity of pore diameter 10-1000 nm. Wang and Ueda observed the increase in the amount of absorbed water and chloride penetration depth in concrete with freeze-thaw damage [6]. The water absorption and chloride penetration occur at a higher rate in damaged specimens compared with undamaged concrete. Chunsheng et al. [7] investigated the impact of mechanical cracking on transport-related properties of concrete. The material damage was quantified using ultrasonic pulse velocity and found that damage increases air permeability significantly, while sorptivity and electrical resistivity were more strongly correlated to the total open porosity. Therefore, the permeability of cement-based material can be used as an important indicator to evaluate durability of structure. The effects of frost degradation on mass transport in damaged mortar specimens by investigating the mechanisms of moisture transport was discussed by Ghasemzadeh et. al. [8]. They observed that mass transport increases with damage irrespective of the measurement method but different methods show different sensitivity to the presence of damage. According to the performed measurements, the water and air permeability increase exponentially with damage and seem to be the most sensitive parameter to the presence of damage. Moreover, initial sorptivity increases linearly with damage.

The purpose of the experimental study was to observe the effect of damage, induced by freeze-thaw loading in mortar specimens, using different mass transport measurement techniques. The influence of frost degradation on oxygen permeability (the intrinsic permeability coefficient) and capillary absorption (the water absorption coefficient) was investigated. The paper presents a study of transport properties carried out on four different cement mortars.

\section{Materials}

\subsection{Mix proportion of cement mortar}

Four mortars made of two type of cement: Portland cement CEM I 42.5R and Blast Furnace Slag cement CEM III/A 42.5 N LH/HSR/NA, with different water-cement ratios were prepared in this study $(\mathrm{w} / \mathrm{c}=0.45$ and 0.40$)$. The fine aggregate consisted of natural quartz sand with a maximum aggregate size of $2 \mathrm{~mm}$. The details of the mixture proportions of cement mortar are given in Table 1 . The specimens were formed in cylindrical molds with a diameter of $50.5 \mathrm{~mm}$ and height of $70 \mathrm{~mm}$, sealed for $24 \mathrm{~h}$ and then demolded. In order to achieve a high degree of hydration and a uniform saturation, the samples were cured in tap water at $20 \pm 1{ }^{\circ} \mathrm{C}$ for 90 days. 
Table 1. Cement mortar mixture proportions.

\begin{tabular}{|c|c|c|c|c|c|c|}
\hline Series & \multicolumn{2}{|l|}{ Cement $[g]$} & Sand $[g]$ & Water $[g]$ & Plasticizer [g] & $\mathbf{w} / \mathbf{c}[-]$ \\
\hline Z_1 & \multirow{2}{*}{ CEM I 42,5R } & \multirow{4}{*}{900} & \multirow{4}{*}{2700} & 405 & 4.95 & 0.45 \\
\hline Z_2 & & & & 360 & 8.10 & 0.40 \\
\hline Z_3 & \multirow{2}{*}{$\begin{array}{l}\text { CEM III/A 42,5N } \\
\text { LH/HSR/NA }\end{array}$} & & & 405 & 4.05 & 0.45 \\
\hline Z_4 & & & & 360 & 8.55 & 0.40 \\
\hline
\end{tabular}

\subsection{Specimen preparation}

After initial conditioning, at the age of 90 days, the top and bottom ends of the cylindrical specimens, roughly $10 \mathrm{~mm}$, were cut away and rejected. Although the cutting of the specimens may result in microcracks close to the cut surfaces, the damage is significantly smaller than the damage due to freeze-thaw which was monitored in the experimental study. Subsequently, the specimens, according to PN-88/B-06250 [9], were subjected to freeze-thaw cycles. A total of 100 freeze-thaw cycles were used and a part of the specimens were also taken from the chamber for testing after 25, 50 and 75 cycles. The analysis of transport properties of specimens made of Blast Furnace Slag cement was extended to 150 freeze-thaw cycles. After the cycling water freezing, the lateral sides of each specimen were insulated by resin and then the cylinders were pasted inside PVC tubes, Fig. 1. Before the measurements, the samples were oven-dried at $60^{\circ} \mathrm{C}$ until mass stabilization and then cooled for $24 \mathrm{~h}$ in a desiccators at $20^{\circ} \mathrm{C}$. The value of applied temperature, due to reduction of large temperature gradients across the specimen, does not produce additional cracking of the cement matrix. Due to the non-destructive nature of tests, all the measurements were carried out on the same specimens $(\varnothing 50 \times 50 \mathrm{~mm})$ at all degrees of damage. After the measurements of the gas permeability were completed, the water absorption coefficient was analysed.

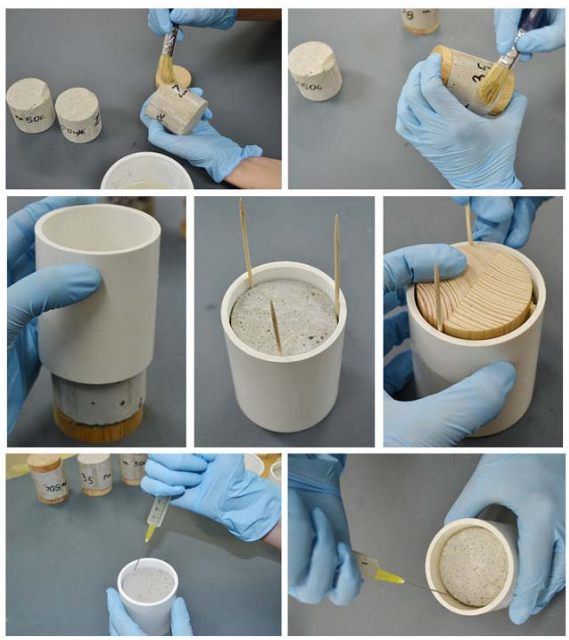

Fig. 1. The stages of sample preparation. 


\section{Methods}

\subsection{Oxygen permeability}

The Cembureau method recommended in $[10,11]$ was adopted to measure oxygen permeability of cement mortars. The self-designed device, in which the PVC tube is one of the elements of the chamber, is presented in Fig. 2. It can be used to simultaneously determine the gas permeability of three specimens.
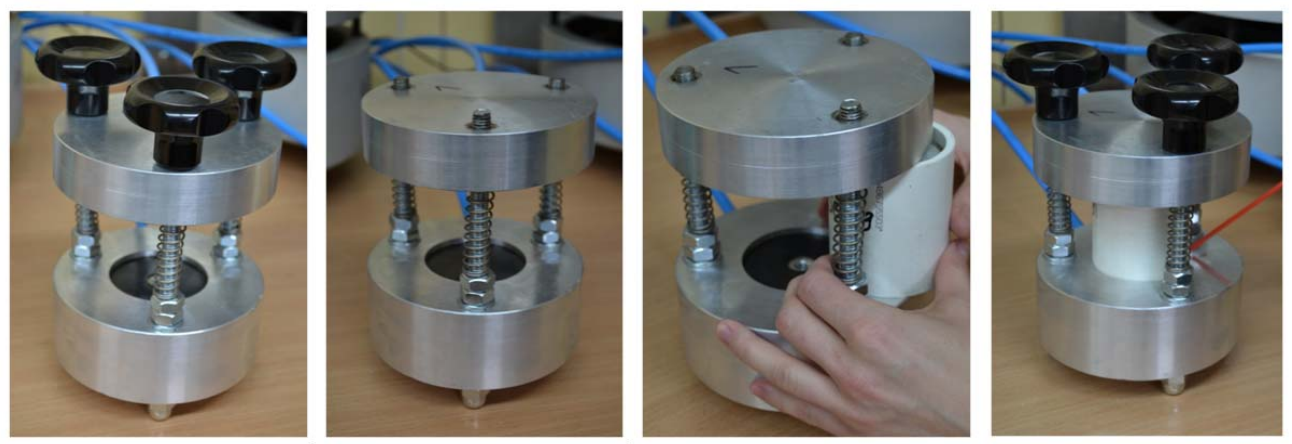

Fig. 2. Device for gas permeability test.

Due to gas pressure gradient, which is induced between two surfaces of samples located in the measuring chamber, a one-dimension gas flow was imposed. The test was based on measuring the volume of oxygen flowing through the specimen within a specified time. The volumetric gas flow meter, calibrated tubes of different volumes from 1 to $100 \mathrm{ml}$, were equipped with a pump which allowed the creation of a soap bubble as an indicator. Before the measurements, steady state flow was established. The volume of single measurements of the flowing gas took between approximately 40 and $60 \mathrm{~s}$. It was assumed, that if two values were separated by a 10 minute time interval differed by less than $3 \%$ the steady state flow condition was achieved. Apparent gas permeability, $k_{a}$, was determined at a given pressure using following equation [10]:

$$
k_{\mathrm{a}}=\left(2 p_{0} Q_{i} \cdot L \cdot \mu\right) /\left[\mathrm{A} \cdot\left(p_{\mathrm{i}}^{2}-p_{0}^{2}\right)\right]
$$

where: $p_{i}$ and $p_{0}$ are the inlet and outlet pressure [Pa], $Q_{i}$ is the volumetric flow rate $\left[\mathrm{m}^{3} / \mathrm{s}\right.$ ], $L$ is the sample thickness in the direction of the gas flow [m], $A$ is the area of the sample cross-section $\left[\mathrm{m}^{2}\right]$, and $\mu$ is the viscosity of oxygen $[\mathrm{Pa} \cdot \mathrm{s}]$. In order to reflect the effects of frost damage on moisture transport in mortar specimens, the measurements of gas permeability were carried out with different degrees of degradation: after $0,25,50,75,100$ and 150 (only specimens made with CEM III) freeze-thaw cycles. All the measurements were conducted for four values of inlet pressure, which were picked depending on the tightness and destruction level of a particular specimen. Subsequently, the obtained values for the apparent permeability, $k_{a}$, were plotted as a function of $1 / p_{m}$, where $p_{m}=\left(p_{i}+p_{0}\right) / 2$ is the mean gas pressure [12]. The permeability parameters: intrinsic coefficient of permeability, $k_{v}$, and slip-flow constant (the Klinkenberg coefficient), $b$, were obtained from a regression analysis as illustrated in Fig. 3. 


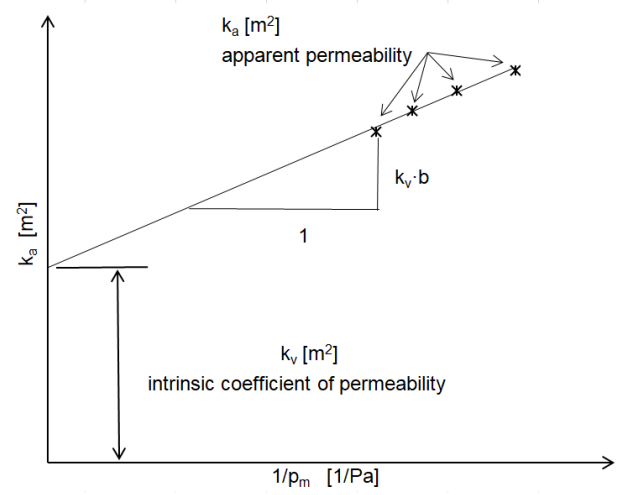

Fig. 3. Determination of permeability parameters: intrinsic coefficient of permeability, $k_{v}$, and slip-flow constant, $b$.

\subsection{Capillarity test}

Standard procedures PN-EN ISO 15148 [13] of determining the water absorption coefficient consisted in weighing samples in given time intervals throughout $24 \mathrm{~h}$ were carried out. The samples were placed in a container filled with water, then the measurements were performed after: 5 and 20 minutes and after: 1, 2, 3, 4, 6, 8, 12 and 24h. The uncontrolled exchange of moisture through the lateral sides with the surrounding was prevented by PVC tube. The water absorption coefficient, $A\left[\mathrm{~kg} /\left(\mathrm{m}^{2} \cdot \mathrm{s}^{1 / 2}\right)\right]$ was determined using a linear relationship between the weight of water which was gained and the square root of time, according to the formula:

$$
A=\Delta \mathrm{m}_{\mathrm{t}} /(F \cdot \sqrt{ } t)
$$

where $\Delta m_{t}$ is the mass increase of the sample [kg] during time $t[\mathrm{~h}]$ and $F$ is the sample surface area in direct contact with water $\left[\mathrm{m}^{2}\right]$.

\section{Results and discussion}

\subsection{Oxygen permeability}

The values of intrinsic permeability of all analyzed samples made of different types of cement and $\mathrm{w} / \mathrm{c}$ ratio after different numbers of freeze and thaw cycles are presented in Fig. 4. The measurements were conducted on three specimens of series $Z \_1$ and $Z \_3$ and four samples of series $Z \_2$ and $Z \_4$ for each number of freeze-thaw cycles. Because of large scatters of obtained values (visible destruction of some mortar samples), the number of specimens for Z_3 after 100 and $150 \mathrm{f}$-t cycles was increased to five.

It was established that gas permeability increased with the rising number of f-t cycles. The frost degradation of the matrix made of Portland cement with a higher value of water to cement ratio was more extensive. The average intrinsic coefficient of permeability of analyzed specimens after the 75th cycle was approximately 40 times higher and at the end of the test it was 100 times higher than the permeability of undamaged specimens. Based on the obtained results, one can noticed that the type of cement had a significant impact on the permeability coefficient of cement mortars exposed to cyclic freezing.

Although, the application of CEM III did not eliminate frost degradation, the ice-induced damage of the cement matrix was consequently reduced. For Z_3 mortar, a significant increase of intrinsic coefficient of permeability could be observed between 
75 and 100 freeze-thaw cycles. For this case the permeability of analyzed specimens after 75 cycles was 2.3 times higher than the reference permeability and at the end of 100 and 150 cycles was approximately 450 times and 3300 times higher than the permeability of undamaged specimens. In case samples made with CEM III with lower water to cement ratio, $\mathrm{w} / \mathrm{c}=0.40$, one can observe that the gas permeability remained rather constant. After $150 \mathrm{f}$-t cycles, the mean value of gas permeability increased with the rising number of f-t cycles only about 6 times.
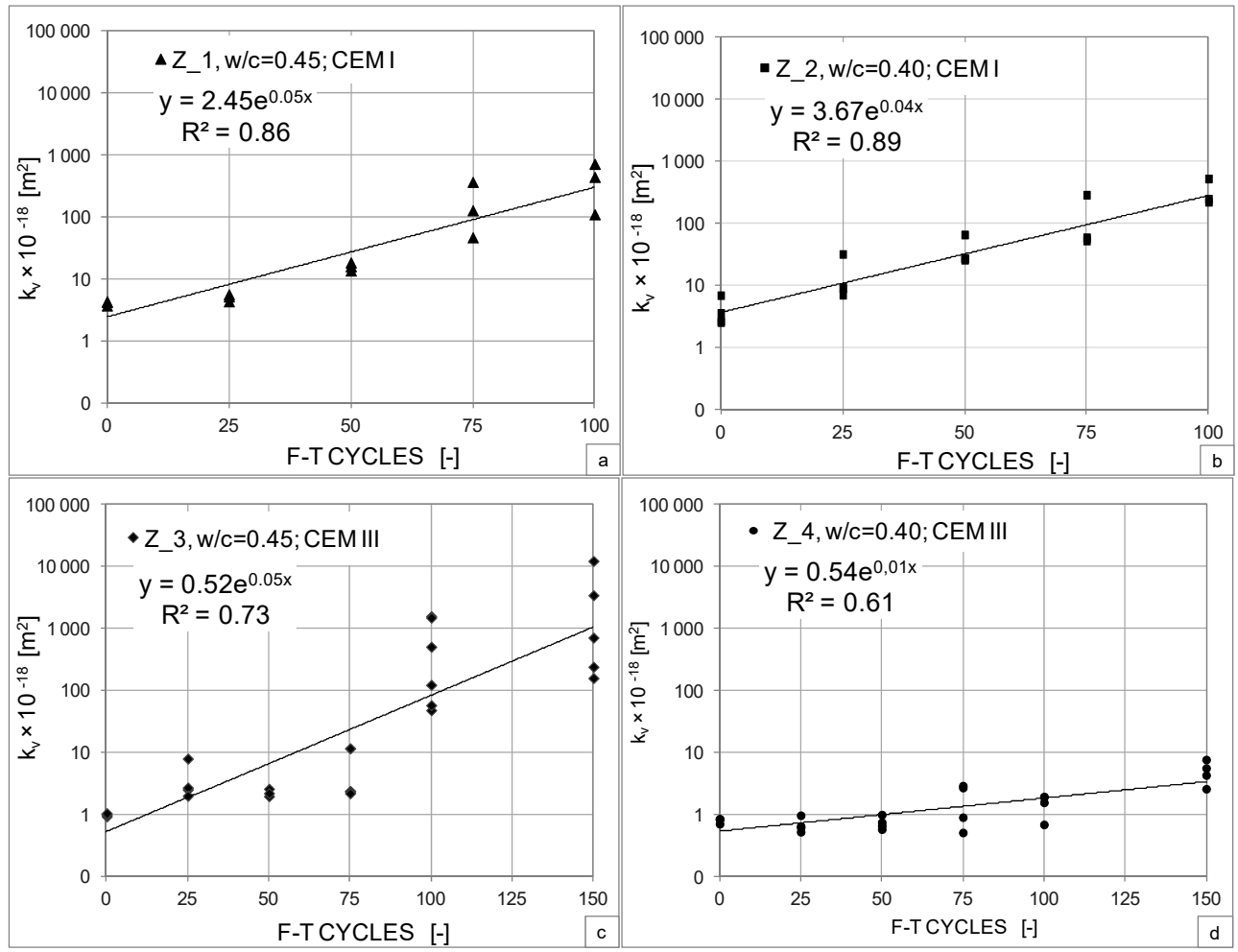

Fig. 4. Intrinsic coefficient of permeability after $n$ freeze-thaw cycles for series $Z \_1$ (a) and $Z \_2$ (b) after $0,25,50,75$ and 100 freeze-thaw cycles and for series $Z \_3$ (c) and $Z \_4$ (d) after $0,25,50,75$, 100 and 150 freeze-thaw cycles.

\subsection{Capillarity test}

The results of the average value of water absorption coefficient after a given number of freeze-thaw cycles, $n$, are presented in Table 2 . The coefficient of variation, $c_{v}$, defined as a ratio of the standard deviation and the mean value of the water absorption coefficient is also shown in Table 2. The gradual ice-induced damage of the cement matrix was confirmed by results of the capillary absorption test. The obtained results showed that the rate of water absorption coefficient gradually increased with the rising number of f-t cycles. Faster water uptake was noticed for the cement mortars with the highest value of $\mathrm{w} / \mathrm{c}$ ratio for which extensive destruction of the matrix was observed (specimens of $Z_{-} 1$ after 75 and $100 \mathrm{f}-\mathrm{t}$ cycles and Z_3 after $150 \mathrm{f}-\mathrm{t}$ cycles). It implies that the microcracks and capillary pores were saturated in a short time. After $24 \mathrm{~h}$ the top of the samples became wet. The specimens with a high level of degradation reached a higher degree of saturation earlier as compared to specimens with a lower level of damage, which is in agreement with other research that studied this problem, i.e. [8]. According to Ghasemzadeh at. el. [8] this 
observation indicates that unsaturated cracks and capillary pores were rapidly filled with water, and then, water diffused into the larger air voids.

The relationship between intrinsic permeability and water absorption coefficient of all tested mortar specimens is shown in Fig. 5a ( $Z_{-} 1$ and $\left.Z_{-} 2\right)$ and $b$ ( $Z_{-} 3$ and $\left.Z_{-} 4\right)$. An exponential relation between the gas permeability, the water absorption coefficient and frost damage coefficient was proposed. The obtained results show that the microcracks had a more significant influence on oxygen permeability and a lesser influence on the water absorption coefficient.

Table 2. The results of capillarity test after $n$ freeze-thaw cycles: $A$ - the water absorption coefficient of the samples which were subjected to $0,25,50,75,100$ and $150 \mathrm{f}$-t cycles, $c_{v}$ - coefficient of variation (ratio of the standard deviation and the mean value of permeability).

\begin{tabular}{|c|c|c|c|c|c|c|}
\hline \multicolumn{7}{|c|}{$A\left[\mathrm{~kg} /\left(\mathrm{m}^{2} \cdot \mathrm{s}^{1 / 2}\right)\right] \pm c_{v}[\%]$} \\
\hline$n$ & 0 & 25 & 50 & 75 & 100 & 150 \\
\hline Z 1 & 0.62 & 0.52 & 0.87 & 1.21 & 1.38 & - \\
{$[\mathrm{w} / \mathrm{c}=0.45, \mathrm{CEM} \mathrm{I}]$} & $( \pm 4 \%)$ & $( \pm 18 \%)$ & $( \pm 16 \%)$ & $( \pm 14 \%)$ & $( \pm 13 \%)$ & - \\
\hline Z_2 & 0.75 & 0.72 & 0.82 & 0.81 & 0.89 & - \\
{$[\mathrm{w} / \mathrm{c}=0.40, \mathrm{CEM} \mathrm{I}]$} & $( \pm 3 \%)$ & $( \pm 7 \%)$ & $( \pm 10 \%)$ & $( \pm 9 \%)$ & $( \pm 4 \%)$ & \\
\hline Z_3 & 0.32 & 0.53 & 0.37 & 0.43 & 0.89 & 1.56 \\
{$[\mathrm{w} / \mathrm{c}=0.45, \mathrm{CEM} \mathrm{III}]$} & $( \pm 6 \%)$ & $( \pm 6 \%)$ & $( \pm 7 \%)$ & $( \pm 17 \%)$ & $( \pm 14 \%)$ & $( \pm 55 \%)$ \\
\hline Z_4 & 0.29 & 0.36 & 0.35 & 0.36 & 0.42 & 0.54 \\
{$[\mathrm{w} / \mathrm{c}=0.40$, CEM III $]$} & $( \pm 12 \%)$ & $( \pm 5 \%)$ & $( \pm 15 \%)$ & $( \pm 9 \%)$ & $( \pm 16 \%)$ & $( \pm 22 \%)$ \\
\hline
\end{tabular}
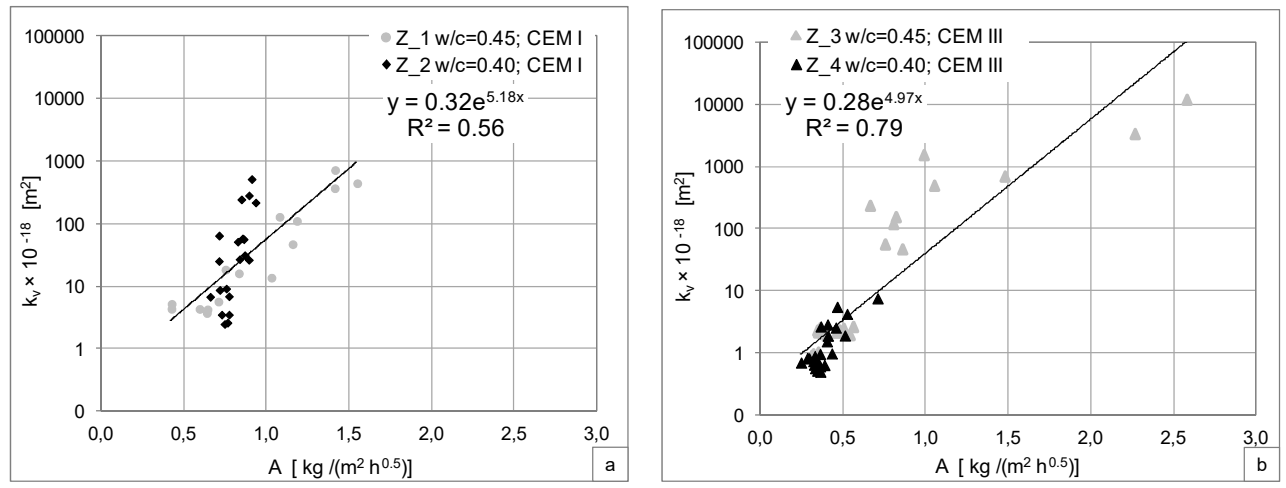

Fig. 5. Relation between intrinsic permeability and water absorption coefficient for series $Z \_1$ and Z_2 (a) and Z_3 and Z_4 (b).

\section{Conclusions}

The performed measurements enabled the estimation of the transport properties of cement mortars subjected to cyclic water freezing. The obtained results allow to conclude that different transport measurement methods showed different sensitivity to frost damage of mortar specimens. The effect of damage changed significantly according to the mechanism of mass transport used in the measurement (flow under pressure gradient or capillary suction). The gas permeability of samples subjected to cycling water freezing increased exponentially, an increase with several orders of magnitude could be noticed. The rapid increase of the intrinsic coefficient of permeability with a growing number of number of freeze-thaw cycles could be caused by the high volume of crack density and increase in crack width. Meanwhile, the water absorption was less sensitive to microcracks. 
The results of measurements also indicate that that type of cement had a significant influence on frost resistance of cement mortar. The positive impact of CEM III on ice-induced cement matrix degradation was visible. However, for a high w/c the application of CEM III did not eliminate frost degradation, the ice-induced damage of cement matrix was consequently reduced. The increase of transport properties, the gas permeability and the water absorption coefficient; was less pronounced until 75 freeze-thaw cycles. Moreover, the samples made of Blast Furnace cement with a lower w/c ratio were more resistant to cyclic water freezing, no structure deterioration could be noticed until 150 frost cycles.

\section{References}

1. J. Kropp, H.K. Hilsdorf, Performance Criteria for Concrete Durability (E\&FN SPON, London, 2005)

2. P. K. Mehta, P.J.M. Monterio, Concrete: Microstructure, Properties, and Materials (Third ed., McGraw-Hill, New York, 2006)

3. Z. Wang, Q. Zeng, Y. Wu, L. Wang, Y. Yao, K. Li, Constr. Build. Mater. 62, 18-27 (2014)

4. A.A. Hamami, Ph. Turcry, A. Ait-Mokhtar, Cem. Concr. Res. 42, 490-498 (2012)

5. J. Zhang, F. Bian, Y. Zhang, Z. Fang, C. Fu, J. Guo, Constr. Build. Mater. 163, 402-413 (2018)

6. L. Wang, T. Ueda, J. Mater. Civ. Eng. 26 (5), 955-965 (2014)

7. Z. Chungsheng, L. Kefei, H. Jianguo, Mater. Struct. 45 (3), 381-392 (2012)

8. F. Ghasemzadeh, R. Rashetnia, D. Smyl, M. Pour-Ghaz, Cem. Concr. Res. 70, 119-129 (2016)

9. PN-B-06250:1988, Polish Standard: Normal Concrete (1988)

10. RILEM Technical Recommendation 116-PCD.1999, Mater. Struct. 32, 174-179 (1999)

11. J. J. Kollek, Mater. Struct. 22, 225-230 (1989)

12. L. J. Klinkenberg, Drilling and Production Practice (API, New York, 1941)

13. EN ISO 15148:2002, Hygrothermal performance of building materials and products Determination of water absorption coefficient by partial immersion (2002) 